\title{
INVERSION OF PHASE TIMES FOR HYPOCENTERS AND SHALLOW CRUSTAL VELOCITIES, MOJAVE DESERT, CALIFORNIA
}

\author{
By James A. Hileman*
}

\begin{abstract}
Phase times for an ensemble of 20 aftershocks of the June 1, 1975, Galway Lake earthquake were inverted to determine hypocenters and local velocity structure simultaneously. A maximum likelihood formulation of linear, leastsquares inversion was used so that the data were weighted according to their estimated variances. A trade-off parameter controlled the relative importance of the RMS error and the amount by which the model changed at each iteration. Individual aftershocks must be selected so that a wide variety of travel paths are represented. Initial trials disclosed that careful constraints are necessary for allowed changes in station delays. Fixed velocity boundaries, based on a priori information, were used for each of several starting models, and only layer velocities were allowed to vary. Each of the trials indicated that shallow crustal velocities in the vincinity of Galway Lake are somewhat lower than those of the usual velocity models. The velocities are not strongly constrained by this data set, but the results are consistent with a subsequent, detailed refraction survey by other workers. Mapping the travel-time surface in time-depth-distance space helps clarify the limitations inherent in a given problem.
\end{abstract}

\section{INTRODUCTION}

A recurring problem in many studies using seismicity data is the accuracy of the earthquake hypocenters. The accuracy of a hypocenter determination depends upon many factors such as the number and spatial distribution of the seismographic stations recording the event, the accuracy with which the onset of phase arrivals can be identified, and the velocity properties of the paths traversed by the phases. The effects of the velocity model have been one of the more persistent problems, particularly in the determination of depths of shallow crustal earthquakes.

This sensitivity of depth to the velocity model comes about when calculated seismic-wave travel times are much less affected by small changes in depth than they are by small changes in horizontal coordinates. The use of nearby stations and $S$-wave arrivals helps constrain the depth estimate. However, in the absence of rigid constraints, a location solution generally can be adjusted to minimize the residuals for any reasonable velocity function, and a depth uncertainty remains.

Further constraints can be included in the problem if an ensemble of different earthquakes is used and the velocity model is allowed to vary also. In this way, we hope to improve the locations, particularly the hypocentral depths, by adjusting the velocity model simultaneously with the locations to fit the travel-time data better. Alternatively, the adjusted velocity model may be the prime objective for some studies. The form of the problem then becomes that of the inversion of arrival times from many earthquakes to simultaneously obtain their locations and the velocity structure in a least-squares sense.

A general development of inversion theory has been given in a series of papers by Backus and Gilbert (1967, 1968, 1970). Their formalism has been modified and applied to crustal velocity estimation by Peters (1973), Aki and Lee (1976), Crosson

* Present address: Fugro, Incorporated, Long Beach, California. 
(1976a, 1976b), Steppe and Crosson (1978), and others. The particular form used here is similar to that of Minster et al. (1974) and Minster et al. (1977) and is modified for the determination of shallow crustal velocities.

In southern California, the varied tectonic provinces lead one to expect that the shallow crustal velocity structure might well be different for each province. The objective of this study was to apply the inversion method to limited areas such as individual aftershock zones or other areas of concentrated seismic activity. The earthquakes used were selected from the aftershock sequence of a 1975 earthquake near Galway Lake in the central portion of the Mojave desert.

\section{MethoD}

For each earthquake, the known quantities are: an initial estimate of the hypocenter location $\vec{m}^{*}=\left(x^{*}, y^{*}, z^{*}, t^{*}\right)$, the locations of $N$ seismographic stations, and the observed arrival times $T_{i}^{0}, i=1, N$. The "forward problem" consists of computing the predicted arrival times $T_{i}{ }^{*}$ using a velocity function appropriate for the area. Later, this velocity function will be considered as a trial velocity function. The initial trial location will include some errors which, in turn, lead to errors in the calculated arrival times. Residual times $\delta T_{i}$ are defined such that $\delta T_{i}=T_{i}^{0}-T_{i}^{*}$ (observedminus-computed) and $\delta \vec{T}$ is the vector of all residuals. It is desired to obtain corrections $\delta \vec{m}=(\delta x, \delta y, \delta z, \delta t)$ to the trial locations so that the residuals for the corrected location, $\vec{m}^{*}+\delta \vec{m}$, will be minimized.

A solution for $\delta \vec{m}$ in the presence of uncertainties in the observed data $T_{i}{ }^{0}$ is obtained following Minster et al. (1974). Let $\delta T_{i}=T_{i}^{0}-T_{i}(\vec{m})$ be the ultimate residuals obtained from the final best location $\vec{m}$. Assume that the $\delta T_{i}$ have independent Gaussian distributions with variances $\sigma_{i}{ }^{2}$. Then from Mathews and Walker (1970), the likelihood function is

$$
L(x, y, z, t)=L(\vec{m})=\frac{1}{(2 \pi)^{N / 2} \prod_{i=1}^{N} \sigma_{i}} \exp \left[-\sum \frac{\left[T_{i}^{0}-T_{i}(\vec{m})\right]^{2}}{2 \sigma_{i}{ }^{2}}\right]
$$

This function is proportional to the probability, or likelihood, that a given set of arrival times $T_{i}^{0}$ will be observed if the true values of the model are $x, y, z, t$. The best model is taken to be the one which maximizes the likelihood function, or is the most likely to have produced the given set of observables. The likelihood function is maximized by minimizing the exponential term. A trial model is introduced and the parameters are assumed to vary smoothly in the vicinity of the trial model so that the equations can be linearized. For details of the derivation, see Hileman (1977) or Minster et al. (1974). The result of the derivation is a system of equations written in matrix form as

$$
A^{T} V^{-1} A \delta M=A^{T} V^{-1} \delta T^{*}
$$

where $A$ is a matrix of all the partial derivatives of arrival times with respect to the various model parameters. $V$ is a matrix of the arrival-time variances, $\sigma_{i}{ }^{2} \delta T_{i}{ }^{*}$ is a vector of the travel-time residuals and $\delta M$ is a vector of differences between the trial model and the best fitting model. We have a least-squares system in which the data are weighted by their variances.

$$
\delta M=\left[A^{T} V^{-1} A\right]^{-1} A^{T} V^{-1} \delta T^{*}
$$


is the solution for the $\delta m$ 's which are then used as corrections to the trial model. The effect of the maximum likelihood formulation is to include the variances of the observed data into the solution and give a rational basis for a weighted least-squares solution.

The likelihood function of equation (1) includes only the location parameters for a single earthquake. Extension of the problem to incorporate many earthquakes as well as parameters of the velocity model consists of enlarging the matrices and vectors appropriately. The $A$ matrix will have only blocks of non-zero terms because all of the earthquakes are independent. There is no relation between the location of one earthquake and the arrival times for any other earthquake. Solution of the problem requires the inverse of the matrix $A^{T} V^{-1} A$. Some of the model parameters, such as hypocenter depths, may be poorly constrained by the data. Their corresponding eigenvalues would be very small, causing instabilities in the numerical matrix inversion and corresponding large fluctuations in the solution vector $\delta \vec{M}$. The simplest method to stablize the matrix inversion is to add a constant to each diagonal term of the matrix; this scheme was given in a more general form by Levenberg (1944). Wiggins (1972) chose to compute all of the eigenvectors and eliminate those having eigenvalues below some arbitrary threshold. The method used here is that of Minster et al. (1977) and is best thought of as controlling the distance in model space that the solution is allowed to move at each iteration. The exponential term of the likelihood function of equation (1) is modified to

$$
F=\cos \theta \sum_{i=1}^{N} \frac{\left[T_{i}^{0}-T_{i}(\vec{m})\right]^{2}}{2 \sigma_{i}{ }^{2}}+\sin \theta \sum_{j=1}^{M} \frac{\left[m_{j}-m_{j}^{0}\right]^{2}}{2 \omega_{j}{ }^{2}}
$$

The $m_{j}$ are the parameters of the current model, $m_{j}{ }^{0}$ are the parameters of a preferred model, and the $\omega_{j}^{2}$ are the allowable variances of the model parameters. The summation in the first term is just the function minimized previously and can be thought of as a measure of the distance in observation space between the observed arrival times and the computed arrival times. The summation in the second term is a similar measure in model space of the distance between the current model and a preferred model. The parameter $\theta$ allows a trade off in the degree to which the solution minimizes the residuals, first term, or the changes in the model, second term. Minimization as before and choosing the preferred model to be the current model leads to

$$
\delta \vec{M}=\left[\cos \theta A^{T} V^{-1} A+\sin \theta W^{-1}\right]^{-1}\left[\cos \theta A^{T} V^{-1} \delta \vec{T}^{*}+\sin \theta W^{-1} \delta \vec{m}^{0}\right] .
$$

The matrix $W$ contains the variances $\omega_{i}^{2}$ assigned to the model parameters. If all the model parameters are independent and given the same variance, this form reduces to the familiar form of adding a constant to the diagonal terms of the matrix to be inverted.

\section{DATA}

The earthquakes used in this study occurred near Galway Lake in the Mojave Desert in June 1975. The main shock of magnitude 5.2 has the coordinates $34^{\circ} 31.12^{\prime}$ $\mathrm{N}, 116^{\circ} 29.56^{\prime} \mathrm{W}, 0138$ hours GMT on June 1. Surface cracks were produced for a distance of $6.8 \mathrm{~km}$ along a fault subsequently named the Galway Lake fault (Hill and Beeby, 1977). The existing U.S. Geological Survey Mojave seismographic network was augmented by portable stations operated near the epicentral area of 
the aftershocks from June 5 through June 15. During that time interval, 95 aftershocks were recorded. Fuis (1976) located all of the shocks of the sequence and constructed many focal-plane solutions. He concluded that the fault was nearly vertical with predominantly right-lateral movement.

Figure 1 shows the setting of the Galway Lake sequence and the distribution of epicenters for the period June 5 through June 15 while the portable stations were in operation. A few foreshocks and the initial aftershocks were in the immediate area of the main shock. Although the trend of the epicenters is similar to that of the surface ruptures, there is a displacement of the epicenters somewhat to the east of the fault trace. The fault-plane solutions indicate planes which dip steeply westward rather than eastward as would be the case if the epicenters and surface ruptures

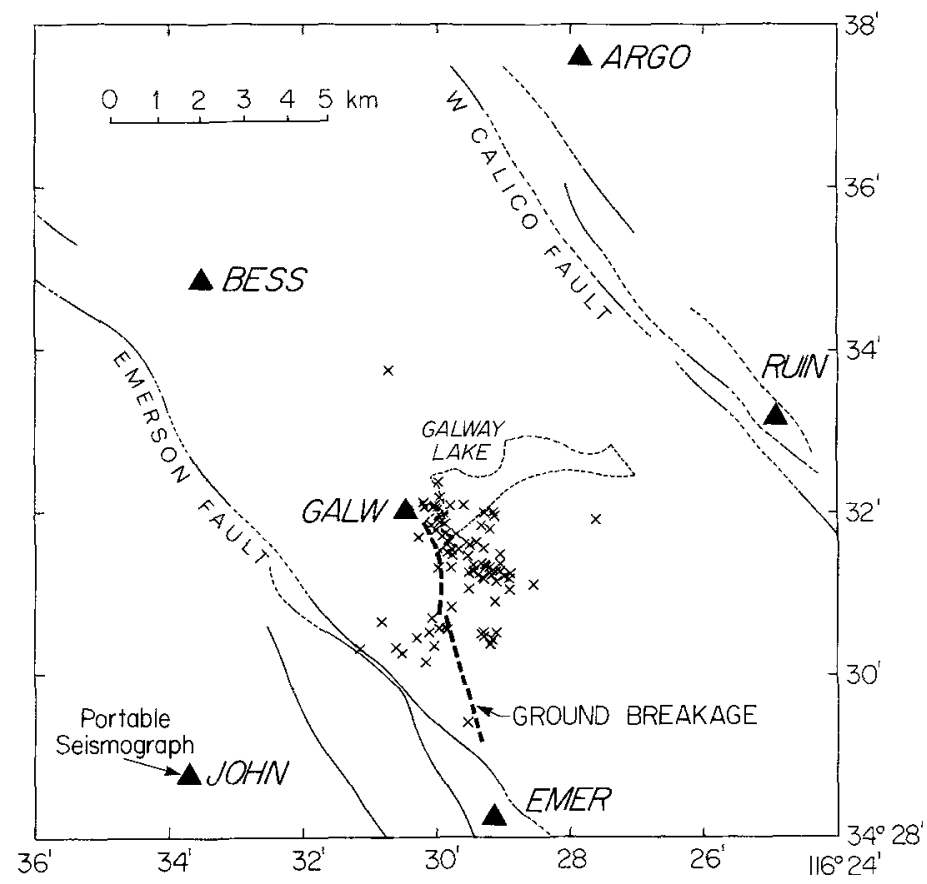

Fig. 1. Aftershocks of the Galway Lake earthquake from June 5 to June 15, 1975, while portable seismographs were operated. Triangles are the station locations.

were consistent. These considerations suggest some systematic bias in the epicenters, such as might be produced by errors in the velocity model used.

Widely scattered, granitic outcrops in the vicinity of Galway Lake indicate a reasonably uniform basement. The major faults are right-lateral, strike-slip with total movement on the order of 10 miles. It is presumed here, but not known for certain, that basement terrains across the faults are similar and that no strong velocity variations occur laterally. With these conditions and inferences, a horizontally layered velocity model seems appropriate for the Galway Lake area.

Although 95 shocks were recorded during the operation of the nearby, temporary stations, not all were suitable for the inversion data set. Some events were discarded because of too few phases, large azimuthal gaps, or obvious location difficulties as indicated by the residuals in their epicenter solutions. The widest possible distribution, both in depth and geographic position, was sought to provide the greatest 
variety in the ray paths. In particular, the selection of two earthquakes with very nearly the same location was avoided. All of these criteria relate to the goal of providing a data set with the greatest possible constraints on the various model parameters. With the above criteria, only about 20 reasonably independent earthquakes could be chosen.

\section{RESULTS AND Discussion}

The hypocenters of 20 selected aftershocks constitute the locations portion of the initial trial model, and the remainder of the model is made up of station delays and the velocity structure. Layer thicknesses were fixed, and only the velocities were allowed to vary. The first trial model, named HYPO71, was based on the station delays and velocity structure used by Fuis (1976) in his study of the aftershock sequence. His velocity structure was determined using refraction data from the Eagle Mountain quarry in the Mojave Desert. The model variances, $\omega_{j}^{2}$ of equation (5), were assigned to be compatible with the assumed precision of location results. Latitudes and longitudes are assumed to be correct within $0.5 \mathrm{~km}$, depths within 1.0 $\mathrm{km}$, and origin times within $0.2 \mathrm{sec}$.

Station delays are common to all 20 events, so a variance of $0.05 \mathrm{sec}$ was used, representing approximately a $\sqrt{N}$ improvement over the origin times. A variance of $0.01 \mathrm{~km} / \mathrm{sec}$ was assigned for the velocities because only small changes to "tune up" the velocities were anticipated.

The results of two problems differing only by the use of slightly different sets of arrival-time data are shown in Figure 2. The vectors show how each epicenter was moved from its starting location; changes in the velocity model are tabulated. For the problem of Figure $2 a$, all of the phase data were used. The problem of Figure $2 b$ used only the $P$-wave data. These is a great difference in the relocation vectors for these two problems. In each case, however, the vectors are essentially parallel and the relative locations have not been changed greatly. Other problems which are not shown, such as using $S$ waves only or using different initial velocities, also gave much the same results but with different vector trends. The cause of these systematic differences was eventually traced to the station delays. The GALW station delay was fixed, as is normally done, so that the delays are relative delays. However, in each case, the stations in the direction of the vector trend were given systematically high delays; those in the opposite direction, systematically low. The sense of these delays is consistent with the observed shifts in epicenters since the delays are subtracted from the observed arrival times. The inversion algorithm was able to trade off between the systematic shift in epicenters and a systematic change in station corrections. After this problem was recognized, constraint equations were added to the system so that the delays could not be biased in any particular direction. Each station-delay correction is a point in latitude, longitude, and delay space. The constraint is achieved by requiring a plane fitting the points in a leastsquares sense to also be horizontal (fixed delay), thereby removing any geographic dependence from the delay corrections.

With the delay-correction constraint in effect, location results for various data sets and starting models became consistent. Figure 3 shows the inversion results for the HYPO71 starting model. The systematic relocation of epicenters westward was a common feature of all solutions after the additional delay constraint was added. Two other velocity model configurations were also used. The initial H-K velocity model was taken from the results of Hadley and Kanamori (1977), and the initial VEL-1 velocity model was simply five arbitrary layers over a half-space. These 

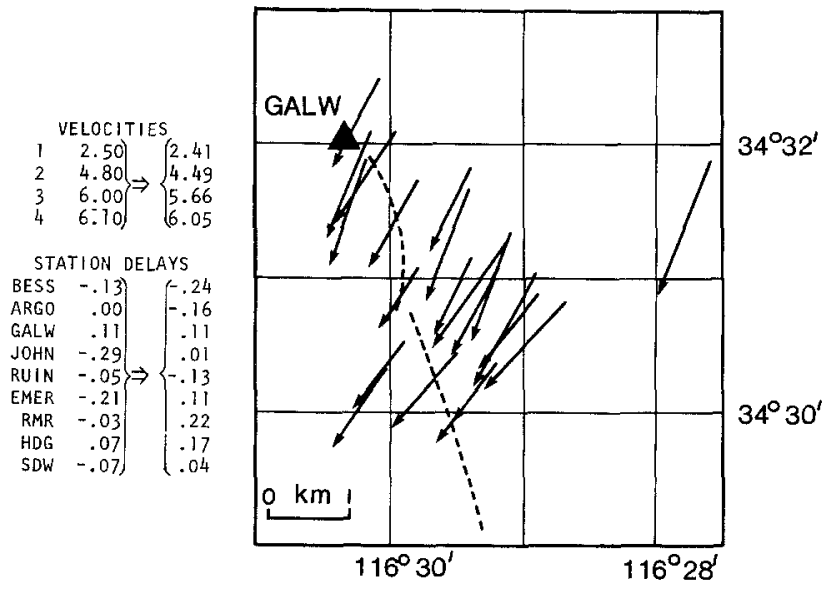

(A)
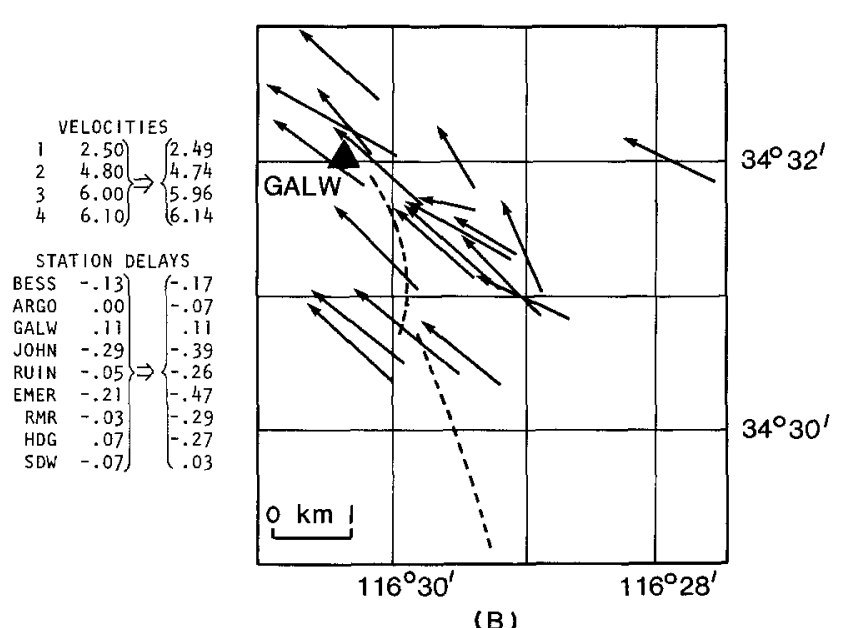

FIG. 2. Inversion results with HYPO71 model before incorporating constraints on station delays. Vectors show changes in the epicenters. The differences between (A) which uses all the phase data and (B) which uses only $P$-waves are attributed to systematic bias introduced into the station delays.

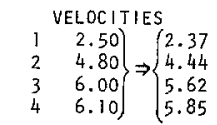

STATION DELAYS

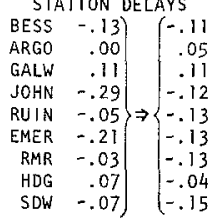

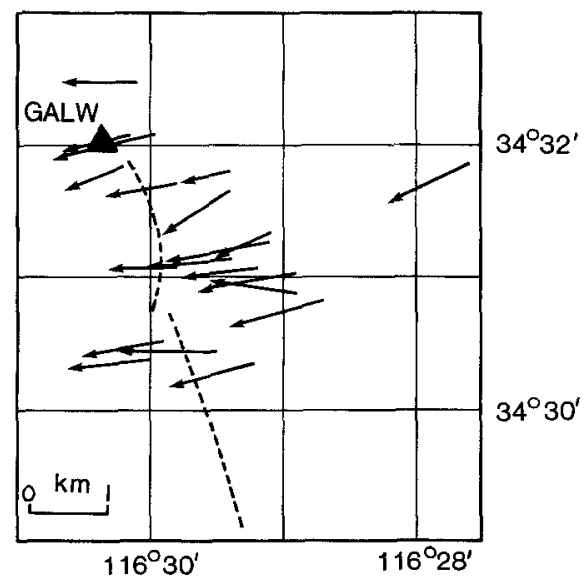

FIG. 3. Inversion results with HYPO71 model when systematic bias in station delays was constrained. 
initial velocity models and the velocities estimated by inversion are summarized in Table 1.

The relocation results were quite similar for all the problems, each estimate being within the error ellipses of the other estimates. In all problems, the velocities estimated by a least-squares fit to the data were consistently lower than those used in the conventional models. However, the velocities were not well constrained by the data and estimates converged rather slowly. Even though one station, GALW, was generally at epicentral distances of a few kilometers, and three additional stations were at distances of 8 to $10 \mathrm{~km}$, most of the shocks were too shallow for their depth estimates to be well controlled. The difficulty in depth control translates directly into difficulties in velocity estimation.

A different insight into some features of the inversion problem comes from considering travel-time surfaces defined by the velocity models. Figure 4 shows the
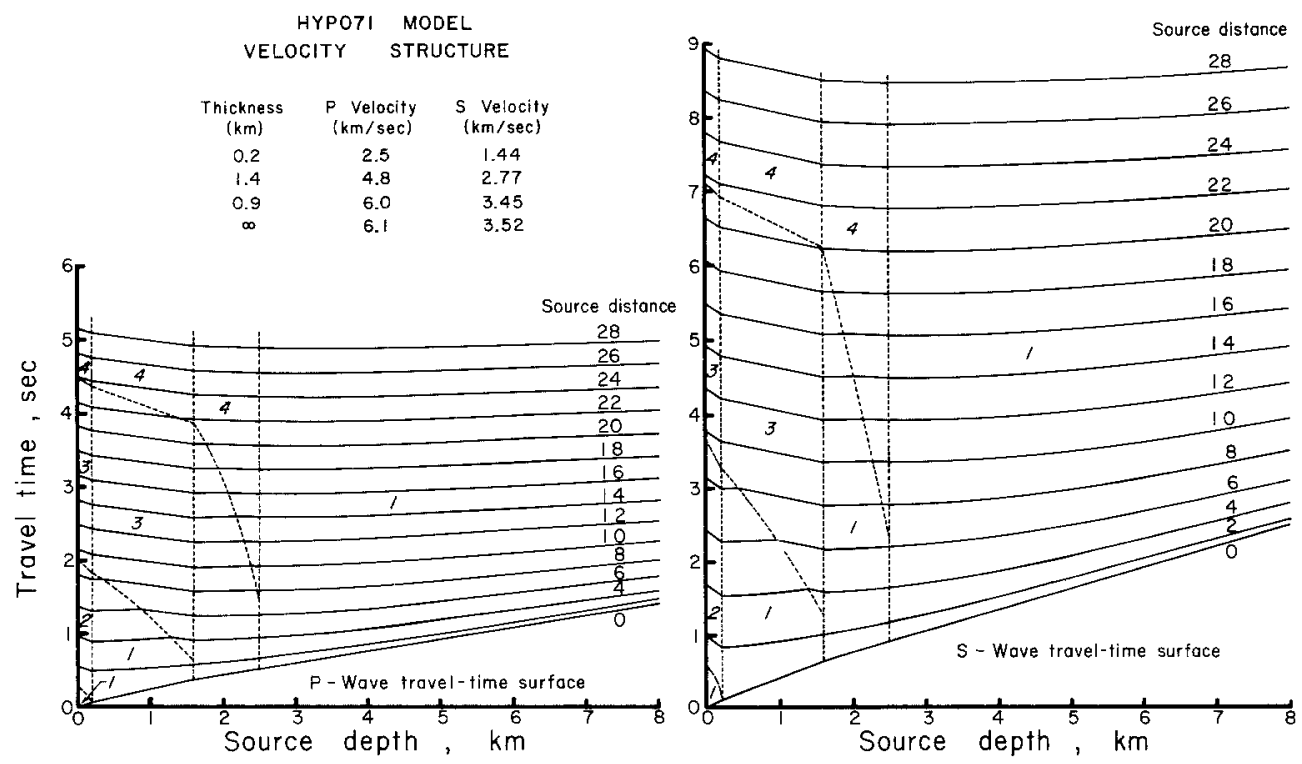

Fig. 4. Travel-time surfaces for $P$ waves and $S$ waves for the HYPO71 velocity model. Contours show travel times as a function of depth for fixed source distances. Slanted numbers indicate types of first arrival; 1, for direct arrival; 2 , for refraction from top of second layer; etc. Dotted lines separate regions with different types of arrivals.

travel-time surfaces for the HYPO71 starting model. The surface is oriented to show travel time as a function of source depth for a number of fixed distances. Each of these curves is a contour line (parallel to the depth-time plane) on the surface. The surface is divided into regions, indicated by numbers and dashed lines, which are defined by the type of ray path that gives the first arrival. Regions labeled 1 have direct arrivals as the first arrival. Regions labeled 2 have a refraction from the top of the second layer as a first arrival, and so forth. These travel-time surfaces illustrate the nature of some of the difficulties in constraining velocities and hypocenter depths. Consider the $P$-wave contour for a distance of $6 \mathrm{~km}$. There is only about $0.1 \mathrm{sec}$ variation in arrival time as the depth is moved between 0.1 and $4.0 \mathrm{~km}$. At other distances, the effect is less severe, but usually a range of very slow change exists. The contours for near distances, 0 to $2 \mathrm{~km}$, clearly show how close-in stations can provide control for depth determination. 

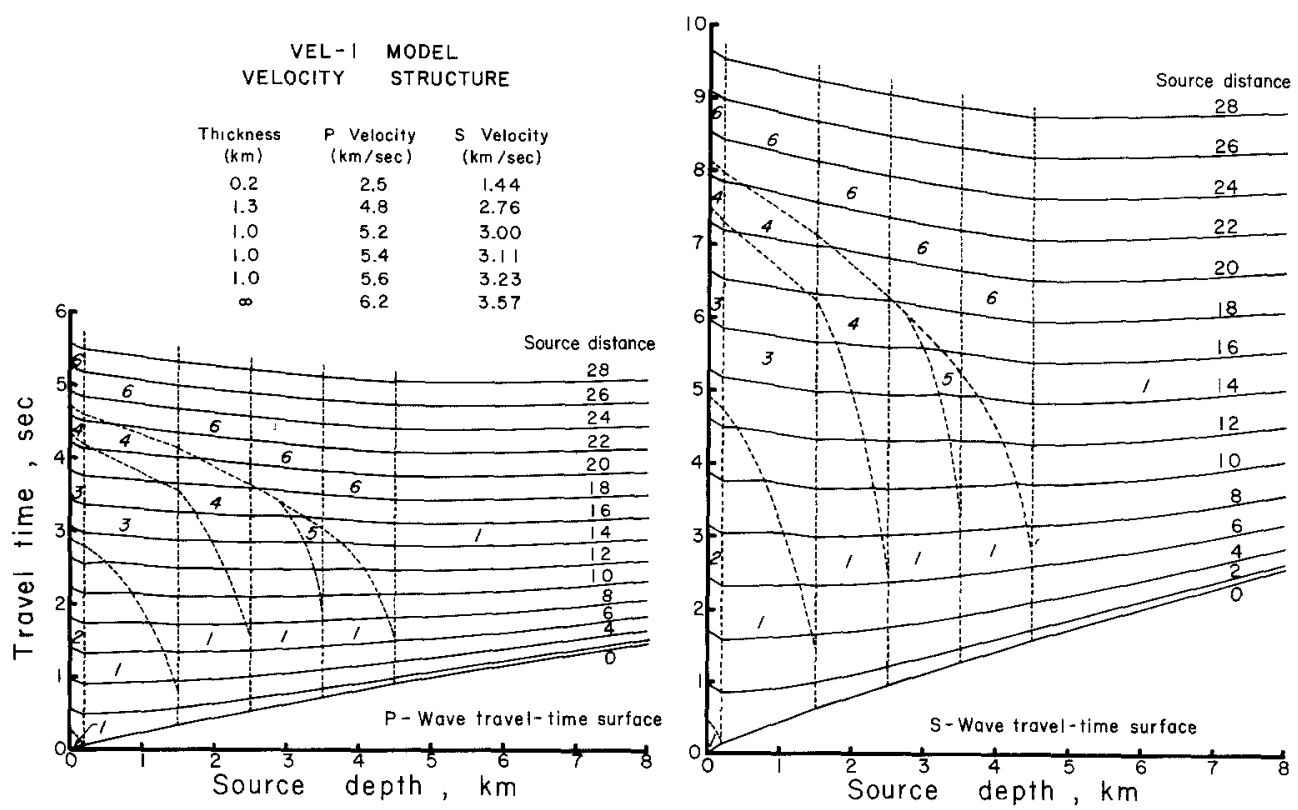

Fig. 5. Travel-time surfaces for $P$ waves and $S$-waves for the VEL-1 velocity model. Notation is the same as for Figure 4.

TABLE 1

SUmmary of Velocity Models and Inversion Results for Galway Lake Data

\begin{tabular}{|c|c|c|c|c|c|c|c|}
\hline Thickness & $\begin{array}{l}\text { Fuis (1977) } \\
\text { Velocity }\end{array}$ & $\begin{array}{l}\text { HYPO } 71 \\
\text { Velacity }\end{array}$ & Thickness & $\begin{array}{c}\text { Hadley and } \\
\text { Kanamori } \\
\text { (1977) } \\
\text { Velocity }\end{array}$ & $\begin{array}{c}\mathrm{H}-\mathrm{k} \\
\text { Velocity }\end{array}$ & Thickness & $\begin{array}{l}\text { VEL-1 } \\
\text { Velocity }\end{array}$ \\
\hline $0.2 \mathrm{~km}$ & $2.5 \mathrm{~km} / \mathrm{sec}$ & $2.4 \mathrm{~km} / \mathrm{sec}$ & $0.2 \mathrm{~km}$ & $2.5 \mathrm{~km} / \mathrm{sec}$ & $2.4 \mathrm{~km} / \mathrm{sec}$ & $0.2 \mathrm{~km}$ & $2.4 \mathrm{~km} / \mathrm{sec}$ \\
\hline 1.4 & 4.8 & 4.5 & \multirow{4}{*}{4.3} & \multirow{4}{*}{5.5} & \multirow{4}{*}{4.9} & 1.3 & 4.5 \\
\hline \multirow[t]{5}{*}{0.9} & 6.0 & 5.6 & & & & 1.0 & 4.8 \\
\hline & \multirow[t]{4}{*}{6.1} & \multirow[t]{3}{*}{5.8} & & & & 1.0 & 5.1 \\
\hline & & & & & & 1.0 & 5.7 \\
\hline & & & & 6.2 & 6.0 & & 6.0 \\
\hline & & $M S=.139$ & & & $\mathrm{RMS}=.143$ & & RMS $=.138$ \\
\hline
\end{tabular}

Lines show the velocity layers for each model at the same scale.

Each arrival time is a point in the coordinate system of the travel-time surface. When locating a single earthquake using a given velocity function, the array of points representing the earthquake is moved to provide a least-squares fit to the travel-time surface. If the range of the points lies completely within one of the refraction regions, the array could be moved freely within the region without changing the fit. This property demonstrates the indeterminancy present if the data 
consist of only refractions from one layer. For the inversion problem with many earthquakes, there is a "cloud" of points to move about, and we also adjust the velocity surface for a better fit. If we can move the points about with some freedom because the depths are poorly constrained, conversely, we can adjust the travel-time surface with corresponding freedom. The geometrical constraints on epicenters provided by having stations well distributed in azimuth are not present in this representation of the travel-time surface. A higher dimensionality to include az1muths would be required.

Figure 5 shows the travel-time surfaces for the VEL-1 velocity model. These surfaces are more complicated because there are more layers in the model, but the general properties are much the same as those for the HYPO71 surfaces. For the distance and depth ranges of the Galway Lake data, we have only weak constraints on the depths and velocities. An interesting aside is that arrivals from layer 5 can be observed only for a limited range of source depths. We have a layered structure with monotonically increasing velocities, yet layer 5 would not be observed in a normal refraction survey.

\section{Conclusions}

The velocity results from three inversion problems using the Galway Lake data are shown in Table 1. Two of the starting models are conventional velocity models for the region. In each case, the velocities estimated by a least-squares fit to the data are lower than those of the usual regional models. However, the velocities were only poorly constrained by the data set. The data are sufficient to indicate that lower velocities provide a better fit to the observations, but not sufficient to distinguish between various layer structures. Subsequent to this study, Fuis (personal communication) did a refraction survey of Galway Lake and his preliminary results seem to indicate that lower velocities are appropriate for shallow depths in this area.

The travel-time surfaces show that a much better data set for the purpose of estimating shallow crustal velocities would result if several of the recording stations were very close to the epicenters. Recording sites should be distributed within the aftershock zone rather than just surrounding it. A station distribution which provides a set of random source distances, rather than a uniform distance, is a better strategy because it provides a greater variety of travel-time paths.

\section{ACKNOWLEDGMENTS}

J. B. Minster and D. L. Anderson have contributed many helpful comments and suggestions. This research was supported primarily by U.S. Geological Survey Contract 14-08-0001-166603, Support of Southern California Seismic Arrays.

\section{REFERENCES}

Aki, K. and W. H. K. Lee (1976). Determination of three dimensional velocity anomalies under a seismic array using $P$-arrival times from local earthquakes: Part I-a homogeneous initial model, $J$. Geophys. Res. 81, 4381-4399.

Backus, G. E. and J. F. Gilbert (1967). Numerical applicaton of a formalism for geophysical inverse problems, Geophys. J. 13, 247-276.

Backus, G. E. and J. F. Gilbert (1968). The resolving power of gross earth data, Geophys. J. 16, 169-205.

Backus, G. E. and J. F. Gilbert (1970). Uniqueness in the inversion of inaccurate gross earth data, Phil. Trans. Roy. Soc. London, Ser. A, 266, 123-192.

Crosson, R. S. (1976a). Crustal structure modeling of earthquake data 1. Simultaneous least squares estimation of hypocenter and velocity parameters, J. Geophys. Res. 81, 3036-3046. 
Crosson, R. S. (1976b). Crustal structure modeling of earthquake data 2. Velocity structure of the Puget sound region, Washington, J. Geophys. Res. 81, 3047-3054.

Fuis, G. S. (1976). Ground breakage and aftershocks of the $M_{L}=5.2$ Galway Lake earthquake, June 1975, Mojave Desert, California (abstract), Trans. Am. Geophys. Union 57, 954.

Hadley, D. M. and H. Kanamori (1977). Seismic structure of the Transverse Ranges, California, Bull. Geol. Soc. Am. 88, 1469-1478.

Hileman, J. A. (1977). Part I: A contribution to the study of the seismicity of southern California, Part II: Inversion of phase times for hypocenters and shallow crustal velocities, Ph.D. Thesis, California Institute of Technology, Pasadena, California.

Hill, R. L. and D. J. Beeby (1977). Surface faulting associated with the 5.2 magnitude Galway Lake earthquake of May 31, 1975: Mojave Desert, San Bernardino County, California, Bull. Geol. Soc. Am. 88, 1378-1384.

Levenberg, K. (1944). A method for the solution of certain non-linear problems in least squares, Quant. Appl. Math. 2, 164-168.

Mathews, J. and R. L. Walker (1970). Mathematical Methods of Physics, Ed. 2, p. 391, W. A. Benjamin, Inc., New York.

Minster, J. B., T. H. Jordan, P. Molnar, and E. Hains (1974). Numerical modeling of instantaneous plate tectonics, Geophys. J. 36, 541-576.

Minster, J. F., J. B. Minster, M. Trevil, and C. J. Allegre (1977). Systematic use of trace elements in igneous process, Part II, Inverse problem of the fractional crystallation process in volcanic suites, Cont. Mineral Petrol. 61, 49-77.

Peters, D. C. (1973). Hypocenter location and crustal structure inversion of seismic array travel times, Ph.D. Thesis, University of Washington, Seattle.

Steppe, J. A. and R. S. Crosson (1978). P-velocity models of the southern Diablo range, California, from inversion of earthquake and explosion arrival times, Bull. Seism. Soc. Am. 68, 357-367.

Wiggins, R. A. (1972). The general linear inverse problem: implications of surface waves and free oscillations for earth structure, Rev. Geophys. Space Phys. 10, 251-285.

SEISMOLOGICAL LABORATORY

California Institute of Technology

Pasadena, California 91125

Manuscript received October 10, 1978 